\title{
Hispanismes
}

Revue de la Société des Hispanistes Français

$18 \mid 2021$

Murs, barrières, obstacles dans les mondes hispaniques II

\section{Les imparfaits du futur}

Humanisme et post-humanisme dans Cielos de la Tierra, de Carmen Boullosa

Futuros imperfectos. Humanismo y posthumanismo en Cielos de la Tierra, de Carmen Boullosa

Imperfect Futures. Humanism and Posthumanism in Carmen Boullosa's Cielos

de la Tierra

\section{Marie-Caroline Leroux}

\section{(2) OpenEdition}

\section{Journals}

Édition électronique

URL : https://journals.openedition.org/hispanismes/14757

DOI : 10.4000/hispanismes. 14757

ISSN : 2270-0765

Éditeur

Société des Hispanistes Français

Référence électronique

Marie-Caroline Leroux, « Les imparfaits du futur », HispanismeS [En ligne], 18 | 2021, mis en ligne le 31 décembre 2021, consulté le 30 janvier 2022. URL : http://journals.openedition.org/hispanismes/14757 ; DOl : https://doi.org/10.4000/hispanismes. 14757

Ce document a été généré automatiquement le 30 janvier 2022

Les contenus de cette revue sont mis à disposition selon les termes de la Licence Creative Commons Attribution - Pas d'Utilisation Commerciale - Pas de Modification 4.0 International. 


\title{
Les imparfaits du futur
}

\author{
Humanisme et post-humanisme dans Cielos de la Tierra, de Carmen \\ Boullosa \\ Futuros imperfectos. Humanismo y posthumanismo en Cielos de la Tierra, de \\ Carmen Boullosa \\ Imperfect Futures. Humanism and Posthumanism in Carmen Boullosa's Cielos \\ de la Tierra
}

Marie-Caroline Leroux

¡Qué raro pensar que de los tres tiempos en que hemos dividido el tiempo - el pasado, el presente,

el futuro-, el más difícil, el más inasible, sea el

presente! El presente es tan inasible como el punto. Porque si lo imaginamos sin extensión, no existe; tenemos que imaginar que el presente aparente vendría a ser un poco el pasado y un poco el porvenir. Jorge Luis Borges, « El tiempo »

1 Carmen Boullosa explore volontiers les potentialités de la fiction historique, et la veine dans laquelle s'inscrivent nombre de ses romans les apparente à ce que la critique a coutume de désigner comme la "métafiction historiographique ${ }^{1}$, ou, dans le cadre plus spécifique de l'Amérique hispanique, le «nouveau roman historique $»^{2}$. Encline à une vision critique du discours historique, la métafiction historiographique en légitime l'incorporation, la récupération ou l'altération dans le cadre romanesque, pour configurer des récits visant à contrer les versions hégémoniques de l'Histoire. Concrètement, la métafiction boullosienne se décline sous des modalités très diverses et nullement exclusives les unes des autres : réécriture de l'histoire officielle, relations de commentaires avec la diégèse ou violations de la logique temporelle.

2 Dans Cielos de la Tierra ${ }^{3}$, ce sont trois époques et trois narrateurs qui dialoguent. Avec la voix d'Hernando de Rivas, indigène lettré $\mathrm{du} \mathrm{XVI}^{\mathrm{e}}$ siècle, $\mathrm{C}$. Boullosa convoque le passé colonial du Mexique. La voix du présent, celle d'Estela Díaz, se manifeste plus 
sporadiquement. Le propos, vagabond et capricieux, tient pour l'essentiel à l'histoire personnelle et familiale de la narratrice. Dans ces chapitres, elle ébauche un dur portrait du Mexique des années 90, insistant au passage sur la part de responsabilité des générations qui l'ont précédée, et la sienne propre, dans le maintien de rapports sociaux et raciaux hérités de la période coloniale. Par l'intermédiaire de la voix de Lear, enfin, on entre dans le champ de la fiction prospective, avec le dessin d'une ère postapocalyptique qui met en scène le peuple des Atlantes, lointain héritier des humains.

3 Le texte d'Hernando, traduit du latin au castillan par Estela, et postérieurement transcrit par Lear pour être archivé au cœur de la Centrale d'Études Avancées, où les siens entreposent les témoignages relatifs à la civilisation terrestre éteinte, fait office de trait d'union entre les différentes trames. Les trois instances qui conduisent la narration alternent au fil des quarante sections qui composent le roman. Le renoncement au continuum chronologique, figuré dans la distribution apparemment aléatoire de la parole entre les narrateurs et dans les va-et-vient temporels qu'elle engage, modifie l'appréhension des temporalités du roman. Pour le dire autrement: le principe rhapsodique met à mal la tripartition du champ temporel, qui est au cœur de l'organisation $d u$ récit autant que de notre perception ordinaire du temps ${ }^{4}$. L'assignation des récits à une époque donnée - passée, présente ou future - devient presque secondaire, et l'on est d'autant plus tenté d'envisager les trois dimensions comme co-extensives que les expériences du temps qui y sont proposées sont étonnamment similaires : nourrie d'une mémoire individuelle et collective traversée par le souffle de l'utopie, chacune vient buter sur un présent en forme d'impasse.

4 À l'issue de ce rapide examen des temporalités de Cielos de la Tierra, oublions un instant ce que l'inscription d'une bonne part du récit dans le futur a de troublant, pour constater que sont ici à l'œuvre un certain nombre de dispositifs habituels de la métafiction et que la cession de la parole à des sujets marginaux engage dans les lignes du présent et du passé une révision critique du discours colonial et du discours de la modernité triomphante qui sont de nature à confirmer l'inscription du roman dans le champ de la métafiction historiographique. Avec cette Histoire à rebrousse-poil le lecteur habituel de $\mathrm{C}$. Boullosa navigue donc en eaux relativement familières.

5 Reste le déploiement de l'imaginaire temporel en direction du futur. L'impression d'étrangeté qu'il produit est atténuée par le fait que, comme souvent chez l'auteure, on se trouve là face à un objet romanesque qui fait la part belle au tribut et se nourrit d'une riche tradition générique : on se rappellera l'intertextualité cervantine dans $L a$ otra mano de Lepanto ${ }^{5}$ ou le souvenir du roman de piraterie dans Son vacas, somos puercos ${ }^{6}$. La littérature d'anticipation sera, pour sa part, l'un des grands arrière-plans de Cielos de la Tierra. Témoin d'une inclination pour l'hybridité propre à l'esthétique postmoderniste, qui n'empêche pas la manifestation de certaines spécificités génériques (la ligne du passé est ainsi traitée sur un mode archaïsant, et hésite entre la chronique et les mémoires), le roman l'est donc aussi du décloisonnement de la littérature science-fictive à l'œuvre ces dernières années.

Le récit de Lear s'inscrit dans un sous-genre particulier de la science-fiction, celui de la littérature post-apocalyptique: la civilisation atlante s'est bâtie sur les ruines de la civilisation humaine, après une catastrophe nucléaire massive. La chute est ici double, car en s'efforçant de ne pas reproduire les erreurs de l'humanité, les Atlantes s'engagent, avec la Réforme du langage, dans un processus qui les conduira eux aussi à leur perte. Il y a de nombreux précédents à ce genre de schéma catastrophiste dans la 
science-fiction contemporaine. On pourrait même remonter plus loin et se rappeler un récit précurseur en la matière : la nouvelle "L'Eternel Adam ", attribuée à Jules Verne. Elle convoque elle aussi l'Atlantide et décrit un monde en proie à de successifs effondrements civilisationnels, qui entraînent l'humanité dans une dramatique spirale de régression. Le constat du narrateur y présente d'étonnantes similitudes avec celui de Lear en termes d'involution de l'espèce ${ }^{7}$. Les contours du corpus auquel puise l'auteure n'apparaissent pas avec clarté mais on songe par ailleurs inévitablement, à la découverte de la barbare utopie atlante, aux dystopies que la fiction d'anticipation contemporaine traîne dans son sillage : à Fahrenheit 451, pour le sort qui y est fait à la mémoire, notamment livresque ${ }^{8}$, à Babel-179 ou The Lenguages of $P a 0^{10}$ pour la réflexion sur l'articulation entre langue et pensée, et bien sûr à $1984^{11}$, où l'État asservit les individus en restreignant les limites de la pensée par une langue appauvrie à l'extrême : le «novlangue $»^{12}$.

7 Ces préalables établis, posons les termes de la réflexion. La relation de l'homme au temps se négocie entre deux contraires : mémoire et oubli. Tout l'enjeu de Cielos de la Tierra tient dans ce tiraillement, et plus précisément, dans le rapport de chaque époque aux temps qui l'ont précédée. La charpente du roman y trouve sa justification. D'un côté du spectre, se tiendront Hernando, Estela et Lear, obsédés par la trace mnésique ; de l'autre, les Atlantes, qui s'arrachent à la marche du temps pour s'abandonner à un présent pur, anhistorique. Avec les Atlantes, Carmen Boullosa donne un visage à l'humain augmenté que la pensée trans-humaniste appelle de ses vœux. Dans la personne de ces êtres immortels dotés de singulières aptitudes, se réalise en effet le dessein de transcender la condition humaine pour l'acheminer vers la «transhumanité $»^{13}$. A ceci près que l'anéantissement de la mémoire auquel ils se livrent se révèle à terme promesse de sauvagerie.

Ce que véhicule la mémoire dans cette œuvre, c'est avant toute chose un legs culturel, que l'on désignera comme « humaniste » faute d'un mot réellement approprié. On sait la flexibilité du terme d'«humanisme", et les problèmes de définition qu'il pose. Il m'arrivera de l'envisager comme cette tradition qui puise ses racines dans la Renaissance et, prenant l'homme comme mesure du monde, promeut un idéal assis sur l'amour de la connaissance et l'élan émancipateur de la pensée. Toutefois, le roman ne place pas tant la question de l'humanisme sur le terrain de la centralité de l'homme que sur celui de la centralité de la connaissance. Si bien que l'humanisme tel que je l'entendrai ici sera plus souvent un rapport à la culture de l'ordre de celui que revendiquait Edward Said dans son ouvrage testament lorsqu'il évoquait la nécessité pour lui de «se protéger et [...] se prémunir contre l'oblitération du passé $»^{14}$. De ce point de vue, le roman est avant toute chose l'histoire d'une défaite. Défaite annoncée dès la ligne du passé, avec l'enterrement du projet éducatif dont avait bénéficié Hernando, qui participait de l'élan renacentista. Défaite manifeste dans la ligne du présent, où l'avènement d'une société du loisir, matérialiste et hédoniste, fait de la mort de l'humanisme bien davantage qu'un simple horizon spéculatif, et enfin dans la ligne future, où l'abolition de la connaissance, du langage et de l'esprit participent d'un dessein de dilution radicale de l'homme dans la nature.

9 L'auteure, donc, impulse un mouvement pendulaire entre ces deux pôles de tension que sont l'humanisme et le " post-humanisme ». Des définitions de ce deuxième terme, aux contours encore problématiques malgré l'abondante littérature qu'il suscite aujourd'hui, je retiendrai d'une part, parce que la ligne du futur s'y rattache, celles qui 
caractérisent le post-humanisme comme un courant visant, après ce qu'il est convenu de désigner comme la "crise de l'humanisme » ${ }^{15}$, à repositionner l'homme dans une relation déhiérarchisée avec son environnement ${ }^{16}$. Mais dans la mesure où la fiction cohabite rarement avec la rigueur définitoire, je lui préférerai souvent un posthumanisme au sens lâche et qu'en matière de relation au passé on pourrait rattacher à l'oubli. Il y a vingt ans de cela, Peter Sloterdijk décrétait dans un essai polémique l'obsolescence de l'humanisme et l'avènement, avec la culture de masse, d'une société qui relèguerait la littérature au rang de "subculture» et dont les bases seraient "résolument post-littéraires, post-épistolaires et, par conséquent, post-humanistes " ${ }^{17}$. C'est sur cette acception du post-humanisme que je mettrai l'accent, au vu de l'obsession pour la désaffection de la mémoire culturelle et du livre qui habitent le roman.

10 Cielos de la Tierra sera donc étudié au filtre de la menace pesant sur la mémoire culturelle et sur les valeurs humanistes qu'elle véhicule. Menace qui s'exprime avec une spéciale acuité dans la ligne du futur. Pour comprendre les enjeux mobilisés par cette question, il nous faudra en passer par une analyse de la relation au passé qui structure le roman, ce pourquoi on commencera par examiner la tessiture des temporalités dans chacune des trois lignes, puis l'attelage des trois récits. On pourra ensuite se pencher sur la transmission hasardeuse de la mémoire dans les temps tourmentés que le roman donne à voir, et qui semblent autant d'imaginaires de notre présent.

11 Pour l'heure, examinons le rapport au temps qui caractérise les trois âges du récit, que l'on envisagera individuellement, avant de les mettre en perspective. Chacun de ces monologues reconfigure une expérience temporelle singulière, subjectivée par le locuteur ${ }^{18}$, et autorise la manifestation d'un temps figuré dans son dépliement interne : Hernando, Lear et Estela se perdent dans les méandres de leur mémoire intime - temps vécu et reconstruit.

12 Le manuscrit d'Hernando prend la forme d'un testament. Cette "fiction d'archive ${ }^{19}$ répond à une visée contre-historique manifeste. Le personnage lègue à la postérité des témoignages décousus de la déchéance de son peuple et le récit de son existence, assis sur la remémoration d'une enfance remontant au premier tiers du XVI ${ }^{e}$ siècle. C'est là un parcours semé de renoncements et de déconvenues, qui témoigne à chaque instant de la violence du fait colonial.

Le récit rend notamment compte du naufrage d'un projet emblématique de l'humanisme missionnaire en Nouvelle Espagne: l'expérience éducative que les Franciscains menèrent à partir de 1536, au sein du Collège de Santa Cruz de Tlatelolco. Le destin d'Hernando épouse celui du projet, les préjugés et les circonstances politiques l'empêchant de prendre l'habit comme il l'aurait souhaité. Obnubilé par le souci de laisser une trace de l'utopie éducative avortée qui a scellé son existence, Hernando commence la relation sous les auspices de l'«anamnēsis » aristotélicienne ${ }^{20}:$ «debo apegarme a la historia que me he propuesto contar ${ }^{21}$. Ce qui ne l'empêche pas de s'abandonner souvent à la survenue du souvenir passif - de la «mnèmē » -, en sorte que ce récit rétrospectif échappe à la stricte linéarité chronologique. À ces échappées belles de la mémoire, il faut ajouter la tension entre le passé auquel il voudrait tout entier se vouer et un présent résiduel mais tenace. Car les circonstances de l'écriture impriment leur marque sur le récit, à travers l'insertion de pseudo-méditations ou de textes en náhuatl, scories destinées à tromper ceux qui lisent par-dessus son épaule. Dans la ligne d'Hernando, se trouvent donc conjugués le souvenir et le temps de sa 
ressaisie et de son écriture. Le jeu métatextuel contribue à maintenir le lecteur dans cet entre-deux : en traitant le récit d'Hernando en archive, en commentant les variations de la calligraphie ou les alternances espagnol/náhuatl, les notes d'Estela nous renvoient constamment, elles aussi, au temps de la production du texte.

Lear et Estela sont dépositaires de ce texte, dont les transcriptions et traductions sont autant de reconfigurations d'une mémoire dont elles aspirent à irriguer le présent. Le récit d'Estela Díaz sera donc pour sa part présenté par la narratrice comme une introduction à sa traduction-adaptation des mémoires d'Hernando. Ici, chaque séquence est un univers, traversé par l'obsession du texte d'Hernando à la fois que sustenté par la trajectoire vitale de la narratrice. Les sujets sur lesquels porte le manuscrit sont le moteur des réminiscences qui mordent et envahissent l'espace narratif. Estela reprend ainsi les thèmes du racisme et de l'échec d'un projet de coexistence raciale alternatif posés par Hernando. L'époque qui lui a échu ne génère en elle que répulsion et elle dénonce les impostures du grand récit national, en mettant à découvert la survie des structures coloniales dans le Mexique de la fin du $\mathrm{XX}^{\mathrm{e}}$ siècle :

Considero que [el manuscrito] de algún modo es mío [...]. Porque soy mexicana y vivo como vivimos los mexicanos, respetuosa de un juego de castas azaroso e inflexible, a pesar de nuestra mencionadísima Revolución y de Benito Juárez y de la demagogia alabando nuestros ancestros indios. Y porque, creo, nuestra historia habría sido distinta si el Colegio de la Santa Cruz de Tlatelolco no hubiera corrido la triste suerte que tuvo. El manuscrito me importa, me concierne como un asunto personal, tal vez también porque mis papás fueron a infestar con el dios único a un pueblo que conservaba hasta entonces, todavía, algún resquicio sano, y me siento responsable de este pecado... O porque mi abuela y sus parientes [...] juzgaron normal hasta hace poco que los indios los llevaran en andas para no llenarse de lodo los zapatos, y porque su familia [...] siempre se casó entre sí con tal de no contagiarse con sangre india $[. ..] .^{22}$

Le récit d'Hernando était avant tout exploration d'une mémoire singulière. Dans le cas d'Estela, la polarisation du souvenir est multiple, comme si l'auteure voulait embrasser par son entremise toute l'histoire du Mexique contemporain. La narratrice, en effet, mêle ses souvenirs avec ceux de ses parents et grands-parents. Avec ce qu'elle désigne comme « estos pasados " ${ }^{23}$, elle compose une fresque historique ponctuée tantôt par un extravagant inventaire gastronomique, tantôt par des allusions aux tragiques métamorphoses des espaces naturels de son enfance. À un présent odieux et à un passé peu amène, Estela préfère toutefois la plongée dans l'ailleurs textuel du manuscrit autographe d'Hernando. L'entreprise dans laquelle elle se lance touche au devoir de mémoire :

Me siento culpable porque pequé al soñar. No soñé, ni yo ni mi generación, con un sueño que borrara la estructura suicida de nuestro pasado colonial. Yo reparo mi pena de la mejor manera : me aplico a traducir del latín al español el texto de un indio $[\ldots]^{24}$.

16 La relation de Lear à son histoire personnelle est bien différente de celle d'Hernando et Estela. Car, conçus artificiellement et élevés en incubateur, les trente-neuf Immortels de L'Atlantide sont aussi dépourvus d'une mémoire infantile propre :

Durante la Conformación, nos transmitieron en el Receptor un sinnúmero de imágenes de nosotros mismos, como éramos ya y como se podía conjeturar seríamos de adultos. Estas imágenes fueron fijas y móviles, planas y de tres dimensiones. En ellas se nos vio en los más dispares paisajes, visitando lugares de la Tierra con la apariencia que tuvieron antes de ser destrozados por el hombre de la Historia ${ }^{25}$. 
17 A la différence de ses congénères, Lear ressent la nécessité de compenser cette carence constitutive, à l'origine de sa vocation d'archéologue. Son récit, impulsé par la découverte du texte d'Estela et de la traduction de celui d'Hernando, est contenu dans le temps de l'instauration de la Réforme du langage. Il se place, au début, dans un temps subjectivisé par la médiation d'une conscience immortelle, étrangère à l'inventaire du passage des heures. L'Atlantide est un univers défini par le collectif, dans lequel les efforts des membres de la communauté convergent vers une fin unique : reconstituer la nature détruite par les hommes. Son état de perfection imminent le livre presque tout entier au présent. Le récit de Lear est, dans les premières sections, en conformité avec les descriptions de l'utopie telle qu'elle est donnée à voir dans les textes canoniques du genre: dans ses évocations du quotidien des Atlantes, Lear use de temps verbaux propres à traduire l'éternité à laquelle ils sont voués. Le présent d'énonciation y cohabite avec des imparfaits installés dans une durée sans terme. Pour le reste, quand elle recourt au prétérit simple, c'est essentiellement pour circonscrire le champ de la genèse de la communauté.

18 À ce point, cependant, l'utopie écologique des Atlantes - leur "écotopie », pour la désigner de quelque manière - reste à finaliser, la Réforme du langage à venir étant considérée par tous comme son véritable point d'orgue. Les étapes de sa mise en place sont rapportées sur un mode quasi simultané dans le journal de Lear, qui commence à coucher par écrit ses réticences et à prendre note des incidents qui accompagnent le processus. C'est ainsi que la mise en place progressive du nouveau code de communication se transforme en moteur insidieux de la réactivation du cours du temps. Là encore, le texte s'en fait l'écho dans l'usage des temps verbaux, la narratrice mobilisant de manière de plus en plus appuyée un prétérit indéfini qui exprime, cette fois, la fin de la stase ${ }^{26}$. Dans les dernières sections écrites de sa main, l'emploi de ce temps verbal change encore: de nouveau, comme dans les premières sections, le prétérit y renvoie à un passé lointain, mais son utilisation sert maintenant un dessein en tous points opposé à sa finalité première puisque, dans son aspect, il désigne un procès achevé qui ne renvoie plus à l'inauguration de l'utopie mais bien à sa clôture :

En la última comunidad de hombres y mujeres, todos fueron iguales, nadie hizo menos al otro por razón de raza, sexo o apariencia. Nadie fue rico ni pobre, poderoso[sic] o esclavo. Se vivió en armonía, se venció la enfermedad, la vejez y la muerte. En esa comunidad idílica que pudo ser eterna, el horror al pasado impuso la destrucción de la especie ${ }^{27}$.

19 Un changement similaire s'opère avec les temps non bornés (présent et imparfait de l'indicatif) ou le passé composé : à partir de la Réforme, ces temps verbaux fréquemment flanqués d'adverbes ou de locutions adverbiales à valeur résultative, signalent la radicalité d'une mutation qui, une fois tous ses compagnons rendus à un état de brutale animalité, condamne Lear à une solitude sans terme ${ }^{28}$.

Ces trois récits mémoriels habités par un impératif moral de transmission sont en définitive pareillement lacérés par le présent: même le temps suspendu de l'utopie atlante est rattrapé par la tension entre l'hier et l'aujourd'hui.

21 Après cette plongée dans les mémoires qui habitent le roman, centrons-nous sur le dessin qui se dégage de l'enchaînement des trois époques et de la dynamique interne de chacune d'entre elles. Si l'on noue les trois fils, la vision du devenir historique qui anime Cielos de la Tierra apparaît avec clarté. Pièce maîtresse du dispositif romanesque, le récit futuriste est significativement celui qui fait montre de la plus grande noirceur 
dans le dessin de la marche de l'Histoire. L'application par les Atlantes de leur projet d'extinction de la mémoire, en refermant l'horizon de l'Histoire, détermine in fine, le sens même de son mouvement : «En esa comunidad idílica que pudo ser eterna, el horror al pasado impuso la destrucción de la especie $»^{29}$.

On trouve une illustration particulièrement prégnante de ce mouvement entropique avec le processus de virtualisation du monde qui se fait jour dans le passage de la ligne du présent à celle du futur. Chez les Immortels, l'image s'impose à la matière et elle médiatise les relations avec le monde environnant: «desde la fundación de nuestra colonia se creyó en el poder de la imagen ", affirme Lear ${ }^{30}$. L'existence des Atlantes est, on l'a vu, assise sur des souvenirs factices destinés à pallier la disparition d'un monde détruit par l'Homme. Cette virtualité quasiment dépourvue de référent partage beaucoup avec l'hyperréalité de Jean Baudrillard, ce déferlement du «simulacre » dont il fait le symptôme de la postmodernité31. Dans un premier temps, Lear dénonce cette fabrique du souvenir comme une imposture : « No lo vivimos, Ramón, tú y yo no fuimos al Cañón, no vimos los camellos. Tenemos que saber que esos recuerdos no son verdad $\|^{32}$. Mais à ce stade, il existe encore une réalité sensible en dehors de ce mensonge. Tel n'est plus le cas dans les derniers temps de l'utopie atlante, qui voit le réel lui-même frappé de déréalisation : Lear, soudain, se dédouble et se voit agir, et des objets apparaissent et disparaissent sous ses yeux. «Las cosas», dit-elle, « suceden, pero no quedan, no se fijan, no permanecen. No son completamente reales $»^{33}$. Cet ébranlement du monde sensible se traduit aussi par un discours convulsif et informe. Tout se passe comme si Lear échouait à circonscrire la nouvelle nature des Atlantes et à assembler les scènes absurdes auxquelles elle assiste.

La notion de « simulacre » héritée de J. Baudrillard a connu une fortune égale à celle de "société du spectacle» développée par Guy Debord ${ }^{34}$, et l'on voit souvent dans le premier un continuateur du second, auquel il fait d'ailleurs parfois référence pour poser, de manière plus radicale, que

[1]a virtualité est autre chose que le spectacle, qui laissait encore place à une conscience critique et à une démystification [...]. Alors que nous pouvions affronter l'irréalité du monde comme spectacle, nous sommes sans défense devant l'extrême réalité de ce monde, devant cette perfection ${ }^{35}$.

Si l'on envisage le roman dans la diachronie, c'est bien une bascule de cet ordre qui paraît s'esquisser entre le texte d'Estela et celui de Lear. Image vivante du grenier de la mémoire, la première séquence d'Estela assemblait des photographies mentales disparates, associées, dans son discours, au discours abrutissant des médias. Le dialogue avec l'imaginaire bon marché de la société de consommation y culminait avec la projection dans une fantaisie née du souvenir des conseils d'une grand-mère obsédée par la préservation de la blancheur de sa peau :

Las imágenes contiguas cambian abruptamente. El lado de la abuela anuncia, con letras enormes, Crema Teatrical, sobreponiéndolas a un rostro femenino, hermoso y sonrosado, sobrepuesto a su vez a la luz tenue del sol naciente cayendo en el volcán, la mujer blanca, el Iztaccíhuatl. El lado de la nieta anuncia con las mismas letras Nivea, sobre el fuego azul de la hoguera, cada vez más azul, hasta volverse el liso y brillante de la tapa ${ }^{36}$.

Estela se trouve ainsi dépossédée d'une mémoire intime irrémédiablement phagocytée par le lieu commun, qui annonce les extrêmes auxquels en arrivera la civilisation atlante, dans la génération de souvenirs artificiels. Du présent au futur, du spectacle au simulacre, l'expulsion du réel est en marche. 
Le pessimisme inhérent à cette poétique de l'Histoire trouve également à s'illustrer dans la dynamique interne de chacune des trois trames, qui pointent, chacune à leur manière, l'effondrement des utopies de leur temps ${ }^{37}$. Cette dynamique dément donc elle aussi toute idée de progrès et inscrit résolument le roman dans le paradigme postmoderne. Il importe de rappeler ici qu'aucun des présents diégétiques n'est statique. Tous, même celui de l'Atlantide, sont des temps de mutation, qui dessinent individuellement ce que l'on pourrait désigner comme un peorvenir, le futur de ces présents étant dépouillé de toute promesse. La courbe des trois récits, pris individuellement, ne fait au reste que confirmer l'implacabilité de la mécanique historique, déjà affichées dans le discours surplombant de la note liminaire, fort de sa prétention de réalités ${ }^{38}$.

Observons la ligne du futur. Avec les Atlantes, on l'a dit, C. Boullosa reprend un axe important de la pensée trans-humaniste, qui pose que l'espèce humaine n'est en réalité que l'ébauche de celle qu'elle pourrait être dans le futur. Si on la resitue dans le débat qui oppose aujourd'hui les «bioprophètes » et les «biocatastrophistes " ${ }^{39}$, force est de constater que la posture pour laquelle opte C. Boullosa est des plus pessimistes. L'enjeu, donc, est celui de la modification du statut ontologique de l'être humain. Dans le roman, l'extinction de ce que fut l'humanité et l'avènement d'une nouvelle espèce, celle des Atlantes, est une donnée qui, comme telle, ne donne guère lieu à commentaire. Ce qui est plus intéressant, c'est l'« amélioration » de leur condition à laquelle procèdent les Atlantes, puisque, pour compléter la Réforme du Langage et atteindre la perfection à laquelle ils aspirent, ils s'amputent la partie du cerveau qui règle la parole et renoncent à l'ouie. Cela, au nom de leur revendication d'appartenance à un ordre plus vaste : la Nature. Là encore, la proposition boullosienne résonne avec force dans le temps présent, et rappelle les postures d'une certaine écologie radicale, qui pousse l'anti-anthropocentrisme jusqu'à la misanthropie, au point de poser la nécessité de la disparition de l'humain. Quoiqu'il en soit, si la parole est ce qui bien nous fait hommes, le projet atlante est donc celui d'un « devenir animal ». Cette proposition extrême n'est pas sans rappeler les hypothèses déjà anciennes de philosophes comme Theodor Adorno ou Alexandre Kojève, tous deux disposés à envisager la possibilité d'une fin de l'Histoire et la disparition des spécificités de la nature humaine ${ }^{40}$. Dans Cielos de la Tierra, cependant, ce serein "devenir animal» se mue en un radical «devenir barbare ", réduisant la trans/post-humanité à une monstrueuse inhumanité. La perte de leurs capacités cognitives débouche effectivement à terme, chez les compagnons de Lear, sur un présent étale, ouvert et sans finalité - bref, sur une temporalité proprement utopique, supposément arrachée à l'Histoire -, mais il n'y a nulle harmonie dans cette vie nue : les Atlantes finissent par se dépecer les uns les autres et se livrent à l'anthropophagie.

On constate ici, pour conclure cette deuxième partie, que l'émancipation des anciens modèles historicistes - des métarécits ${ }^{41}$-, passe par leur mise à l'épreuve romanesque : l'amère proposition post-humaniste qui s'incarne dans le récit de Lear coexiste avec l'effondrement de la téléologie chrétienne (Hernando) et du discours euphorique de la raison et du progrès (Estela). Les accents eschatologiques des divagations d'Hernando, marquées par les prophéties évangéliques, cohabitent ainsi avec des questionnements existentiels qui résonnent des tremblements de sa foi vacillante ${ }^{42}$. Dans sa séquence finale, la reprise d'une phrase attribuée par Fray Bernardino de Sahagún aux dignitaires aztèques dans la controverse qui les avait opposés aux religieux envoyés par 
le Pape pointe la perte de la foi sur un mode plus radical : «Si muriéramos, muramos; si pereciéremos, perezcamos ; que a la verdad, los dioses también murieron ${ }^{43}$. Le futur d'Hernando se trouve à terme déserté par toute transcendance :

Soy persona de poca iluminación, lejana a la luz de la fe, perdida en las tinieblas del desprendimiento, el desencanto, el desapego. No le pertenezco a nada [...]. Elevo por la noche mis rezos para que la luz de Dios recubra con luz las tinieblas en que habito ${ }^{44}$.

Pour Lear, la Réforme se paie de «la pérdida del futuro $»^{45}$, et on peut enfin lire, chez Estela : «nos orillamos al silencio, y al peor silencio, peor aún del que hieden sin parar los cadáveres: el silencio que se escucha cuando ha muerto el Sueño ${ }^{46}$. C'est à Estela qu'il incombe de personnifier le désenchantement de l'ère contemporaine. Elle dénonce aussi bien le mythe de l'accès du Mexique post-révolutionnaire à la modernité que la faillite de l'utopie socialiste cubaine. Chez elle, la négation du progrès comme moteur de l'Histoire affecte aussi le discours du capitalisme triomphant, ultime et funeste rempart de l'utopie dans le $\mathrm{XX}^{\mathrm{e}}$ siècle déclinant :

La pobreza creciente [...], la sobrepoblación, la carencia total de oportunidades para las nuevas generaciones, la suspensión de crecimiento económico y el decrecimiento productivo, etcétera, etcétera... Ninguno de los males mencionados parecen expresar del todo el horror que puede sentir la gente de mi generación $[\ldots]^{47}$.

Et il faut aussi compter avec le motif de l'apocalypse, décliné dans les quatre niveaux celui du temps-cadre de la note liminaire et celui des trois récits qui font l'essentiel de la matière romanesque. Cielos de la Tierra est publié en 1997, dans une décennie où la fin du second millénaire dope l'imaginaire apocalyptique, mais on pourrait difficilement accuser l'auteure d'avoir succombé à la tentation descriptive pour ce qui touche à ce sujet. Pour inéluctable que soit le grand désastre final, on en cherchera en vain une image dans ce miroir brisé de l'Histoire qu'est le roman. Comme souvent dans la littérature d'anticipation, l'apocalypse s'y résume à une béance : elle est un événement proprement infigurable.

31 La rumination de ce schéma involutif constitue en tout cas l'écrin idéal à l'expression d'une inquiétude plus concrète : celle qui touche à la fragilité des modèles culturels. Dans les trois lignes est affichée la nécessité d'un passage de témoin. Et dans les trois lignes, ce relais peine à se faire. Et une fois les chaînes de transmission brisées, c'est tout le délicat édifice de la mémoire culturelle qui s'écroule : mémoire des derniers héritiers de la civilisation aztèque ; mémoire de la première génération d'élèves du Collège de Santa Cruz de Tlatelolco, éduqués dans le cadre de l'ambition franciscaine de réaliser une authentique "Iglesia Indiana »; ou mémoire de l'humanité collectée par les Immortels de l'Atlantide. Si elle se porte sur des objets de nature très différente, cette inquiétude relative à la circulation de la mémoire vaut surtout pour le présent, comme on le verra dans la dernière partie de cette étude.

On a en effet jusque-là juxtaposé puis superposé les trois temps du récit, or cet objet romanesque tient en réalité davantage du dispositif stéréoscopique. Et ce qui se dégage de ce kaléidoscope est une image du temps depuis lequel parle l'auteure et qui constitue encore, à peu de choses près, notre présent. C. Boullosa ne dit rien d'autre dans son préambule, si tant est, bien sûr, qu'on puisse faire crédit de la sincérité à une auteure qui affectionne les miroirs et multiplie volontiers les figures auctoriales ${ }^{48}$.

On est par ailleurs d'autant plus tentés d'appréhender le roman comme l'image d'un présent dilaté à l'extrême et tendu à la fois vers le passé et le futur que Cielos de la Tierra 
est adossé à deux genres littéraire qui regardent volontiers le présent au miroir de l'époque qu'ils prennent pour point de référence. Je veux bien entendu parler du roman historique et du roman d'anticipation. Depuis l'essai fondateur de Georg Lukács ${ }^{49}$ jusqu'aux travaux des théoriciens du nouveau roman historique, la littérature critique a suffisamment commenté la capacité du roman historique à proposer une vision critique du présent sous couvert du passé pour qu'il ne soit plus nécessaire d'y insister. La littérature d'anticipation use de biais similaires. Fredric Jameson part d'ailleurs de l'analyse que G. Lukács fit en son temps du roman historique et de son inscription dans une sensibilité propre à la modernité - que l'on pourra résumer dans une vision dynamique de l'Histoire assise sur le progrès - pour mettre en parallèle ces deux genres. Il observe que l'un comme l'autre sont « le symptôme d'une mutation de notre rapport au temps historique lui-même », et que le déclin du roman historique coïncide avec la naissance de la science-fiction, «qui enregistre désormais le sens naissant du futur, et qui le fait dans l'espace même où s'inscrivait jadis [dans le roman historique] le sens du passé ». Il conclut :

[L]'apparent réalisme, l'apparente représentationnalité de la SF, dissimule depuis toujours une structure temporelle bien plus complexe: il ne s'agit pas de nous donner des «images» du futur [...], mais de défamiliariser et de restructurer l'expérience que nous avons de notre présent $[. . .]^{50}$.

En ce sens, donc, les visées du roman historique ne sont guère distinctes de celles du roman d'anticipation.

Ceci étant dit, le déplacement de perspective auquel nous force l'approche sciencefictionnelle en transformant le présent en passé du futur fait de la ligne de Lear celle qui a le plus de force pour ce qui touche au discours sur la fin de l'humanisme en ce que, précisément, elle est invitation à regarder le présent dans lequel nous sommes pris, et que nous peinons à penser, comme le lieu de la genèse des mutations à venir. Si l'hyperbole est un ressort habituel des dystopies littéraires, les problématiques de l'Atlantide se prêtent au reste facilement à l'actualisation. Nous ne sommes pas là face à un exemple de science-fiction "réaliste ${ }^{51}$, ne serait-ce que parce que les caractéristiques biologiques des Atlantes les distinguent par essence de la race humaine. Pourtant, la ligne future se fait indéniablement l'écho des grandes obsessions et interrogations de la fin du $\mathrm{XX}^{\mathrm{e}}$ siècle et de ses craintes ou de ses aspirations face à une radicale mutation civilisationnelle, à l'horizon du post-humain.

Le concept de "présentisme » forgé par François Hartog me semble pouvoir contribuer à mieux cerner les caractéristiques de cette mutation pour ce qui touche à l'évolution de notre rapport à la mémoire, qu'il m'intéresse ici de développer. Dans son analyse de l'évolution des "régimes d'historicité » - concept qui engage l'expérience et la représentation humaine du temps -, l'historien pose que se sont succédés, en Occident, différents ordres du temps : à un présent à construire sur le modèle du passé a suivi une vision tendue vers le futur et le progrès, qui a laissé place, depuis la chute du mur de Berlin, à un rapport tout entier médié par le présent, un présent surdéterminé et invasif. Cette «crise du temps» qui caractérise l'époque contemporaine, c'est le «présentisme»:

Aujourd'hui la lumière est produite par le présent lui-même, et lui seul. En ce sens [...], il n'y a plus ni passé ni futur, ni temps historique, s'il est vrai que le temps historique moderne s'est trouvé mis en mouvement par la tension créée entre champ d'expérience et horizon d'attente ${ }^{52}$. 

l'Atlantide sont troublantes, notamment pour ce qui touche à la tentation de la patrimonialisation, symptôme du présentisme aux yeux de l'historien. La muséification du monde d'autrefois à laquelle se livre la communauté des Immortels avec la Centrale d'Études Avancées marque à ce titre l'évidence de la césure d'avec le passé. La seule valeur dont y est doté le livre est celle de la trace, de la relique. Le contenu de ceux qui sont sélectionnés pour intégrer la Centrale est externalisé et dématérialisé - notons que cet état ultime du livre l'apparente à la mémoire numérique qui prolonge déjà l'humanité de l'ère digitale-, mais ils sont devenus lettre morte: nul n'en a plus l'usage. La solution de continuité entre passé et présent devient manifeste avec la Réforme : le déraillement de la chaîne de transmission du savoir prend finalement la forme d'une abjuration radicale de la mémoire et de la culture, la possibilité d'une dissidence reposant sur les épaules de Lear, désormais seule capable de faire résonner les œuvres littéraires du passé dans le triste off-word de l'Atlantide. Cielos de la Tierra épouse en cela le schéma classique des dystopies contemporaines, qui voient émerger des figures de marginaux en rupture avec le modèle de société dominant. Comme dans Fahrenheit 451, L'Atlantide proscrit le livre parce qu'il est une menace pour la sérénité d'un monde qui fait de l'amnésie et de la non-pensée le fondement du bonheur, et comme chez R. Bradbury, un individu prend sur ses épaules le poids de la mémoire : Lear devient femme-livre, au même titre que le personnage de Guy Montag devient le « couvre-livre " ${ }^{53}$ de l'Ecclésiaste.

L'importance de la passation n'est jamais si manifeste dans le roman que pour ce qui a trait à la traduction, qui redonne vie au livre menacé de muséification. Hernando est un survivant, et ce manuscrit qu'il cache dans un pied de chaise se veut, entre autres choses, le réservoir de la mémoire des siens. Traduire son texte du latin à l'espagnol, comme le fait Estela dans le présent, c'est réunir les conditions de possibilité de la circulation d'un document qui sans quoi ne serait accessible qu'à une poignée d'érudits. Le personnage regrette de ne pouvoir le faire en náhuatl, afin de le rendre à ceux à qui il revient et d'éviter que ne sombre un pan de la mémoire culturelle des héritiers du peuple aztèque. La langue savante, langue hégémonique s'il en est, est ainsi devenu ce fossé qui empêche l'accès des indigènes à leur propre histoire. Le labeur de traduction d'Estela rappelle inévitablement le travail réalisé au XVI siècle par Fray Bernardino de Sahagún, qui avait mené à bien une grande partie de ses investigations sur la culture indigène au sein même du Collège de Santa Cruz de Tlatelolco. Les visées de $B$. de Sahagún étaient en premier lieu évangélisatrices, mais son Historia general de las cosas de Nueva España n'en relevait pas moins d'une entreprise de sauvegarde de la mémoire culturelle aztèque, et la confiscation du manuscrit et de ses brouillons atteste du danger que recelait l'œuvre aux yeux la Couronne. Dans un cas comme dans l'autre, se pose la question des enjeux politiques associés à la traduction. L'impossibilité d'Estela à traduire le texte-source d'Hernando vers le náhualtl - son incapacité à le décolonialiser - la mène au constat de son incapacité à participer à une inversion du rapport de force culturel ${ }^{54}$.

Dans un roman qui interroge la possibilité de la transmission en régime de rupture, la mémoire contre-historique n'est pas la seule pour laquelle bataillent les personnages. C'est, plus largement, de la sauvegarde d'une mémoire culturelle adossée à une approche universaliste et généreuse de la connaissance - à une approche humaniste qu'il est question. 
40 La romancière sonde les formes prises par cette mémoire. Entendu comme accomplissement de l'individu par l'éducation et la culture, l'humanisme est assis sur une chaîne de transmission, constitutive de toute forme de tradition culturelle. Hernando, fils de son siècle, est initié au latin, aux arts libéraux et aux fondements de la Scolastique. Son manuscrit est émaillé de références à des théologiens ou des poètes qu'il cite de mémoire, car dans un temps où le livre demeure un bien rare le savoir, cimenté par un répertoire de références partagées, s'appuie sur l'ars memorativa. Le temps passant, l'homme-mémoire cède la place au livre imprimé, qui étend son empire avant de refluer brutalement dans le dernier tiers du $\mathrm{XX}^{\mathrm{e}}$ siècle, remplacé par la mémoire déléguée de l'ère numérique. Le roman en passe, pêle-mêle, par tous les états du livre : le manuscrit, avec Hernando et ses deux continuatrices ; le livre dématérialisé de la Centrale; le livre à l'ère de sa circulation massive avec Cien años de soledad, auquel Estela rend un hommage appuyé, rappelant l'engouement fulgurant de toute une génération pour cette œuvre, dans un moment de floraison exceptionnelle de la littérature hispano-américaine ${ }^{55}$; et enfin le texte mémorisé, qui peut prendre le tour, chez Hernando, de citations extraites de la règle franciscaine, ou, chez Lear, d'une érudition plus éclectique. De la ligne passée à la ligne future, le texte mémorisé fait ainsi retour, confirmant, s'il était besoin, la coextensivité des temps du roman.

41 Si la transmission est une condition de la pensée, celle-ci ne peut véritablement se déployer que dans la confrontation, moteur de l'émancipation intellectuelle et garante de la vitalité du savoir. La dynamique transtextuelle ${ }^{56}$ qui sous-tend Cielos de la Tierra se met ainsi au service du principe de la circulation des idées: le texte d'Hernando n'exhibe pas toujours les cicatrices des réécritures successives que lui imposent Estela, Lear et d'autres encore, mais sa condition palimpsestique éclaire constamment le rôle de la mémoire et celui de l'appropriation, de la recréation et du dépassement qui incombent aux héritiers. On peut ainsi lire, chez Estela :

Él [Hernando] existió, pero él ya no es real. Lo he ido difuminando en mi libre traducción, le he borrado los rasgos a punta de imponerle mis intenciones e ideas, mis expectativas de lo que él debiera decir, de lo que debiera haber dicho ${ }^{57}$.

Construit par l'épreuve du dialogue, le texte de la ligne du passé active divers ressorts métanarratifs. Adresses directes à un lecteur futur ou commentaires du vieil érudit quant à la présence de curieux autour de lui dans le temps de l'écriture, inventaire paratextuel des gestes herméneutiques ou traductifs auxquels se livrent les lecteurs successifs d'Hernando: tout concourt à mettre en avant la circulation entre les composantes de la triade auteur-texte-lecteur qui, conformément aux postulats des théoriciens de la réception, participent de la concrétisation de l'œuvre littéraire. Le propre de Cielos de la Tierra est que l'acte de lecture auxquels se livrent les personnages $\mathrm{y}$ donne lieu à des traductions d'un texte princeps - le fameux manuscrit - dont le produit supposé est la version qu'il nous est finalement donné de lire. Pour le dire autrement, ce qui pourrait n'être qu'une opération mentale se trouve réalisé sous une forme matérielle des plus concrètes. Irriguant le texte premier, les expériences interprétatives cumulées de tous les lecteurs d'Hernando - soit: la somme des opérations traductives et de leurs prolongements exégétiques - le soumettent à d'infinies métamorphoses. Et il nous faut renoncer à la quête d'un hypothétique original pour embrasser cet hypertexte absolu, confirmation de ce que «le temps des œuvres n'est pas le temps défini de l'écriture mais le temps indéfini de la lecture et de la mémoire $»^{58}$. L'im-permanence du texte est signe de la vigueur de l'œuvre. 
le temps du présentisme et de l'oblitération de l'hier, face à l'émergence de nouvelles manières d'être au monde et de potentiels sujets amnésiques ; à l'horizon, en somme, du tournant post-humaniste postulé par P. Sloterdijk, le roman défend donc une vision classique des humanités, avec ces personnages de passeurs attachés à mobiliser la littérature pour expliquer le monde, à se nourrir du passé pour éclairer le présent ${ }^{59}$. À ce titre, Cielos de la Tierra constitue bien une tentative de "fortalecer la posición del sujeto humanista [...] en crisis $»^{60}$. Mais on éprouve toute la fragilité de cette proposition dans les pages finales du roman. Pour échapper à l'horreur du monde qui l'entoure, ne reste en effet d'autre solution à Lear que de se réfugier dans les territoires du livre : «Formaremos una nueva comunidad. / La nuestra se llamará Los cielos de la Tierra $\|^{61}$. L'avenir de l'humanisme repose sur une utopie textuelle qui fait peser sur le lecteur la responsabilité de la mémoire qu'enserre le livre : à nous, donc, de devenir couvre-livre...

\section{BIBLIOGRAPHIE}

ALCÁNTAR, Iliana, « Una lectura posmoderna de Cielos de la Tierra de Carmen Boullosa:

Recuperación de la memoria, la historia y la utopía a través de la escritura », ConNotas. Revista de

Crítica y Teoría Literaria, vol. 3, nº 4-5, 2005.

BAUDRILLARD, Jean, Le crime parfait, Paris, Galilée, 1995.

BAUDRILLARD, Jean, Simulacres et simulation, Paris, Galilée, 1981.

Bouju, Emmanuel, « Exercices des mémoires possibles et littérature “à-présent”. La transcription de l'histoire dans le roman contemporain ", Annales. Histoire, sciences sociales [on line], 2010, $\mathrm{n}^{\circ} 2$ (65 année), p. 417-438 [consulté le 25/05/21] <URL : https://www.cairn.info/revueannales-2010-2-page-417.htm>.

Boullosa, Carmen, La otra mano de Lepanto, México, Fondo de Cultura Económica, 2005.

Boullosa, Carmen, Son vacas, somos puercos, México, Era, 1991.

BoullosA, Carmen, Cielos de la Tierra, Madrid, Alfaguara, 1997.

BRADBURY, Ray, Fahrenheit 451, Londres, Rupert Hart-Davis, 1957 [1953].

BRADBURY, Ray, Fahrenheit 451, Paris, Folio SF, 1995 [1953].

BRAIDotti, Rosi, The Posthuman, Cambridge, Polity Press, 2013.

BRown, J. Andrew, « "Humanismo cyborg”: el letrado posthumano en América Latina », Revista de Crítica Literaria Latinoamericana, Año 34, n 68, p. 20 [consulté le 25/06/2021] <URL: http:// www.jstor.org/stable/25479077>.

CASTRO, Holanda, « Intrahistoria y memoria del presente en Cielos de la Tierra de Carmen Boullosa », in Priscilla GAC-ARTIGAS (éd.), Reflexiones. Ensayos sobre escrioras hispanoamericanas contemporáneas, vol.1, New Jersey (USA), Ediciones Nuevo Espacio, coll. Academia, 2002, p. $163-183$. 
DEBORD, Guy, La société du spectacle, Paris, Buchet-Chastel, 1967.

DELANY, Samuel R., Babel-17, Londres, Gollancz, 2010 [1966].

GENETTE, Gérard, «L'utopie littéraire », Figures I, Paris, Seuil, Essais, 1966, p. 123-132.

GENETTE, Gérard, Palimpsestes. La littérature au second degré, Paris, Seuil, Essais, 2003 [1982].

HaRToG, François, Régimes d'historicité. Présentisme et expériences du temps, Paris, Seuil, La librairie du XXI ${ }^{\mathrm{e}}$ siècle, 2003.

Hutcheon, Linda, A Poetics of Postmodernism: History, Theory, Fiction, New York/Londres, Routledge, University paperback, 1990 [1988].

JAMESON, Fredric, Penser avec la science-fiction, Paris, Max Milo, L’Inconnu, 2008 [2005].

JAMESON, Fredric, Archéologies du futur. Le désir nommé utopie [Archaeologies of the Future: The Desire Called Utopia and Other Science Fictions], Paris, Max Milo, L'Inconnu, 2007 [2004].

LECOURT, Dominique, Humain, post-humain, Paris, PUF, coll. Science, histoire et société, 2003.

LEROUX, Marie-Caroline, «¿La autori(ali)dad en pedazos ? Muerte y resurrección del autor en El complot de los Románticos, de Carmen Boullosa», in Alejandro PALMA CASTRo, et Cécile QUINTANA, (dir.), Deconstruccion del espacio literario en América Latina. 1996-2016, Éditions des archives contemporaines, 2019, p. 51-58 [consulté le 31/12/2021] <URL: http://eac.ac/books/ $9782813003409>$.

LUKACS, Georg, Le roman historique [Der historische Roman], Paris, Payot, 1977 [1956].

LYOTARD, François, La condition postmoderne. Rapport sur le savoir. Paris, Minuit, 1979.

MENTon, Seymour, La nueva novela histórica de la América Latina, 1979-1992, México, Fondo de Cultura Económica, 1993.

ORWELL, George, 1984, New York, Spark Publishing, 2014 [1949].

ORWELL, George, 1984, Paris, Gallimard, Folio, 1991.

REID, Anna, « Cielos de la Tierra, ¿Utopía o apocalipsis? », Espéculo. Revista de estudios literarios, Universidad Complutense de Madrid, 2007 [consulté le 20/02/2022] <URL: https://webs.ucm.es/ info/especulo/numero35/cielosti.html >.

RICœUR, Paul, La mémoire, l'histoire, l'oubli. Paris, Seuil, L'ordre philosophique, 2000.

RIC๕UR, Paul, Temps et récit I. L'intrigue et le récit historique, Paris, Seuil, Points Essais, 1991 [1983].

SAID, Edward, Humanisme et démocratie [Humanism and Democratic Criticism], Paris, Fayard, 2005 [2004].

SÁNCHEZ HERNANDEZ, Diana Sofía, «El sujeto como eje constructor del pasado en Cielos de la Tierra de Carmen Boullosa » [consulté le 31/01/2021] <URL: https://www.lehman.cuny.edu/ciberletras/ v23/sanchezdiana.html >.

SLOTERDIJK, Peter, Règles pour le parc humain. Une lettre en réponse à la Lettre sur l'Humanisme de Heidegger [Regeln für den Menschenpark. Ein Antwortschreiben zu Heideggers Brief über den Humanismus], Paris, Mille et Une nuits, La petite collection, 2000 [1999].

VANCE, Jack, The Languages of Pao, New York, Ace books, 1958.

VERNE, Jules et BENETT, León, MYRBACH-RHEINFELD, Felician von, Roux, Georges (ill.), « L'Éternel Adam », Hier et demain. Contes et nouvelles, Paris, Hachette, 1910. 


\section{NOTES}

1. Voir Linda Hutcheon, A Poetics of Postmodernism: History, Theory, Fiction, New York/Londres, Routledge, University paperback, 1990 [1988].

2. Voir Seymour Menton, La nueva novela histórica de la América Latina, 1979-1992, México, Fondo de Cultura Económica, 1993.

3. Carmen Boullosa, Cielos de la Tierra, Madrid, Alfaguara, 1997.

4. Holanda CASTRO, revenant dans l'étude qu'elle consacre à Cielos de la Tierra sur la prédilection d'une grande partie de la critique pour l'axe « historique » du roman - le temps de la Colonie -, souligne avec justesse le fait que l'organisation tripartite du récit n'est pas qu'une question d'époques : «Aunque es innegable el monumental trabajo que ha realizado Boullosa en cuanto a la época, no podemos obviar la importancia de haber organizado una novela en la medida de tres tiempos, y no sólo de tres épocas, sino [sic] los tres tiempos de la vivencia humana, presente, pasado y futuro » (Holanda CASTRO, «Intrahistoria y memoria del presente en Cielos de la Tierra de Carmen Boullosa ", in Priscilla GAC-ARTIGAS (éd.), Reflexiones. Ensayos sobre escritoras hispanoamericanas contemporáneas, vol. 1, New Jersey, Ediciones Nuevo Espacio, coll. Academia, 2002, p. 167-168. C'est moi qui souligne par l'italique.

5. Carmen BoullosA, La otra mano de Lepanto, México, Fondo de Cultura Económica, 2005.

6. Carmen Boullosa, Son vacas, somos puercos, México, Era, 1991.

7. «Il est, hélas ! trop certain que l'humanité, dont nous sommes les seuls représentants, est en voie de régression rapide et tend à se rapprocher de la brute. [...] On peut dire que notre vie cérébrale est abolie [...].

[A]vec eux, avec nous, ces traces légères des hommes que nous fûmes - car nous ne sommes plus des hommes, en vérité - vont disparaître à jamais [...].

Il me semble les voir, ces hommes futurs, oublieux du langage articulé, l'intelligence éteinte, le corps couvert de poils rudes, errer dans ce morne désert » (Jules VERNE et León BENETT, Felician von MYRBACH-RHEINFELD, Georges Roux (ill.), «L'Éternel Adam », Hier et demain. Contes et nouvelles, Paris, Hachette, 1910, p. 240-243).

8. Ray BRADBURY, Fahrenheit 451, Londres, Rupert Hart-Davis, 1957 [1953].

9. Samuel R. DeLANY, Babel-17, Londres, Gollancz, 2010 [1966].

10. Jack VANCE, The Languages of Pao, New York, Ace books, 1958.

11. George ORWELL, 1984, New York, Spark Publishing, 2014 [1949].

12. Pour la traduction française, voir George oRWELL, 1984, Paris, Gallimard, Folio, 1991 [1949].

13. "Issu d'une concrétion des mots «transition » et " humain » avec une forte connotation de dépassement, le transhumanisme revendique une recherche scientifique illimitée et une application immédiate à l'humain des modifications génétiques, du clonage, de la trangenèse, des nanotechnologies, du couplage du cerveau et de l'informatique, etc. Ce courant idéologique, qui connaît bien des déclinaisons, pousse à son terme une utopie postmoderne qui prend le relais des anciens grands récits pour promettre des lendemains enchantés, et même l'immortalité » (David LE BRETON, « Le transhumanisme ou l'adieu au corps ", Écologie\& Politique, n 55 (2017/2), p. 81-93 [consulté le 31/01/2021] <URL: https://www.cairn.info/revue-ecologie-et-politique-2017-2page-81.htm >.

14. Edward SAID, Humanisme et démocratie [Humanism and Democratic Criticism], Paris, Fayard, 2005 [2004], p. 243.

15. Rappelons les vives critiques dont l'idée de centralité de l'homme et l'humanisme ont pu faire l'objet sur les fronts du marxisme, de la psychanalyse, de la pensée post-coloniale ou du poststructuralisme, tout au long du $\mathrm{Xx}^{\mathrm{e}}$ siècle.

16. Voir Rosi BRAidotTi, The Posthuman, Cambridge, Polity Press, 2013. 
17. Voir Peter SLOTERDIJK, Règles pour le parc humain. Une lettre en réponse à la Lettre sur l'Humanisme de Heidegger [Regeln für den Menschenpark. Ein Antwortschreiben zu Heideggers Brief über den Humanismus], Paris, Mille et Une nuits. La petite collection, 2000 [1999], p. 13.

18. C'est le " temps humain ", tel que le conçoit Paul RICOEUR, in Temps et récit I. L'intrigue et le récit historique, Paris, Seuil, Points Essais, 1991 [1983], p. 215.

19. J'emprunte le terme à Emmanuel Bouju, in «Exercices des mémoires possibles et littérature "à-présent". La transcription de l'histoire dans le roman contemporain ", Annales. Histoire, sciences sociales [on line], 2010, $\mathrm{n}^{\circ} 2$ (65 ${ }^{\mathrm{e}}$ année), p. 417-438 [consulté le 25/05/21] <URL: https:// www.cairn.info/revue-annales-2010-2-page-417.htm>.

20. «L'histoire des notions et des mots est [...] instructive : les Grecs avaient deux mots, mnèmé et anamnèsis, pour désigner d'une part le souvenir comme apparaissant, passivement à la limite, au point de caractériser comme affection - pathos - sa venue à l'esprit, d'autre part le souvenir comme objet d'une quête ordinairement dénommée rappel, recollection » (Paul RICoEUR, La mémoire, l'histoire, l'oubli. Paris, Seuil, L'ordre philosophique, 2000, p. 4).

21. Carmen Boullosa, Cielos, Op. cit., p. 119.

22. Ibid., p. 65.

23. Ibid., p. 48.

24. Ibid., p. 204.

25. Ibid., p. 108.

26. «Cuando estaba por dejar mi habitación de L'Atlantide para bajar a continuar mi transcripción [...], me pasó algo tan enojoso que [...] siento como que me sucede de nuevo »; « un mediodía vi a un atlántido agachado en cuatro patas bebiendo del agua caliza de un estanque podrido » (Ibid., p. 89, p. 265). Italiques ajoutés.

27. Ibid., p. 362. Italiques ajoutés.

28. «Han olvidado ya la lengua y sus poderes »; " No arremedaban al animal ni a la cosa. Eran... Eran imbéciles, atroces... Horrendos... Eran hombres sin alma, no remedos de simios ni de piedras »; "Ya no hay manera de comprenderlos » (Ibid., p. 336, p. 336, p. 313). Italiques ajoutés.

29. Carmen Boullosa, Cielos, Op.cit., p. 362.

30. Ibid., p. 108.

31. Voir Jean BAUDRILLARD, Simulacres et simulation, Paris, Galilée, 1981. J. BAUDRILLARD distingue trois ordres du simulacre : le simulacre de contrefaçon, qui suppose l'existence préalable d'un original ; le simulacre de production industrielle, fruit d'une fabrication en série qui abolit la nécessité de l'original; et enfin un troisième ordre, celui du simulacre de simulation, qui correspond à l'hyperréalité. « Plus vraie que le vrai : telle est la simulation ». Dans un monde où le réel est complètement recouvert sous le virtuel, seule demeure l'illusion : le temps présent est celui du meurtre de la réalité (in Le crime parfait, Paris, Galilée, 1995).

32. Carmen Boullosa, Cielos, Op. cit., p. 115.

33. Ibid., p. 322.

34. Voir Guy DeBoRD, La société du spectacle, Paris, Buchet-Chastel, 1967.

35. Jean BAUDRILLARD, Le crime, Op. cit., p. 49-50.

36. Carmen Boullosa, Cielos, p. 41.

37. Pour ce qui touche aux liens avec l'utopie, voir Anna REID, "Cielos de la Tierra, ¿Utopía o apocalipsis?", Espéculo. Revista de estudios literarios, Universidad Complutense de Madrid, 2007 [consulté le 20/02/2022] <URL: https://webs.ucm.es/info/especulo/numero35/cielosti.html >.

38. «La guerra intercontinental se ha desatado. Si no llegan las potencias a un arrgelo expedito, si no se solucionan las pugnas internas de los territorios en que hubo naciones, pocos meses quedarán al hombre y tal vez a la naturaleza » (Ibid., p. 14).

39. Voir Dominique LeCourT, Humain, post-humain. Paris, PUF, coll. Science, histoire et société, 2003. 
40. A propos de cet horizon posé par $\mathrm{T}$. Adorno, qui consisterait à «vivre comme de bons animaux ", Fredric JAMESON fait le commentaire suivant : "L'utopie, cet abandon de l'instinct de conservation, serait alors cette condition dans laquelle il deviendrait concevable de vivre comme les animaux, dans un pur présent, de vivre dépouillé de toute peur concernant la survie et de toute angoisse de l'avenir, de toute lutte, de toute préoccupation tactique et stratégique - de toute Sorge! -, en un mot de tout ce qui fait l'histoire (ou la préhistoire) humaine : si tout cela venait à disparaître, la "nature humaine" actuelle se verrait remplacer par une autre, encore totalement inconcevable» (in Archéologies du futur. Le désir nommé utopie [Archaeologies of the Future: The Desire Called Utopia and Other Science Fictions], Paris, Max Milo, L'Inconnu, 2007 [2004], p. 300).

41. Voir François LYOTARD, La condition postmoderne. Rapport sur le savoir. Paris, Minuit, 1979.

42. « ¿Seré por siempre sólo hueso y polvo?»(Carmen Boullosa, Cielos, Op. cit., p. 80).

43. Ibid., p. 365.

44. Ibid., p. 364-365.

45. Ibid., p. 323.

46. Ibid., p. 204.

47. Ibid., p. 148.

48. «Si alguna autoridad tengo ante este libro, diré que la verdadera autoría no pertenece a ninguno de los que he dicho, sino al pulsar de una violencia destructiva que percibí en el aire, en mi ciudad y en otros sitios [...] » (Ibid., p. 9). Relativement à la problématisation de la question de l'autorité chez C. Boullosa, voir notamment Marie-Caroline LEROUX, « ¿La autori(ali)dad en pedazos ? Muerte y resurrección del autor en El complot de los Románticos, de Carmen Boullosa», in Alejandro PALMA CASTRO, et Cécile QUINTANA, (dir.), Deconstruccion del espacio literario en América Latina. 1996-2016., Éditions des archives contemporaines, 2019, p. 51-58 [consulté le 31/12/2021] <URL:http://eac.ac/books/9782813003409>.

49. Voir Georg LUKACS, Le roman historique [Der historische Roman], Paris, Payot, 1977 [1956]

50. Fredric JAMESON, Penser avec la science-fiction, Paris, Max Milo, L'Inconnu, 2008 [2005], p. 12, p. 15 et p. 16.

51. La science-fiction réaliste étant entendue comme celle-là qui est « axée sur le futur proche ou fondée sur une perspective dystopique » (Fredric JAMESON, Archéologies, Op. cit., p. 243).

52. François HARTOG, Régimes d'historicité. Présentisme et expériences du temps, Paris, Seuil, La librairie du XXI ${ }^{\text {e }}$ siècle, 2003, p. 217.

53. C'est la traduction pour laquelle opte l'édition française. Voir Ray BRADBURY, Fahrenheit 451, Paris, Folio SF, 1995 [1953], p. 198.

54. La personne du chroniqueur, la teneur de ses propos, l'enjeu que constituent la traduction et la transmission de son récit: tout nous renvoie à la question de la subversion du discours hégémonique de l'histoire, que j'ai brièvement présentée en introduction comme un axe majeur de la métafiction historiographique. Ce n'est pas à cette aune que la présente étude examine Cielos de la Tierra, aussi ne développerai-je pas davantage cet aspect. Pour aller plus loin on pourra consulter, entre autres travaux, ceux d'Iliana ALCÁNTAR et de Diana Sofía SÁNCHEZ HERNANDEZ. Voir Iliana ALCÁNTAR « Una lectura posmoderna de Cielos de la Tierra de Carmen Boullosa: Recuperación de la memoria, la historia y la utopía a través de la escritura ", ConNotas. Revista de Crítica y Teoría Literaria, vol. 3, n 4-5 (2005), et Diana Sofía SÁNCHEZ HERNANDEZ, « El sujeto como eje constructor del pasado en Cielos de la Tierra de Carmen Boullosa» [consulté le 31/01/2021] <URL: https:// www.lehman.cuny.edu/ciberletras/v23/sanchezdiana.html >.

55. Carmen Boullosa, Cielos, Op. cit., p. 198-205.

56. J'emploie ici le terme au sens genettien, comme «tout ce qui [...] met [le texte] en relation, manifeste ou secrète, avec d'autres textes " (Gérard GENETTE, Palimpsestes. La littérature au second degré, Paris, Seuil, Essais, 2003 [1982], p. 7. 
57. Carmen Boullosa, Cielos, Op. cit., p. 145.

58. Gérard GENETTE, «L'utopie littéraire », Figures I, Paris, Seuil, Essais, 1966, p. 123-132, p. 132.

59. «[E]s mi lectura...», dit Estela, « de un manuscrito que me pertenece... que me explica mi presente »(Carmen Boullosa, Cielos, Op. cit., p. 67).

60. J. Andrew BRown, « Humanismo "cyborg”: el letrado posthumano en América Latina », Revista de Crítica Literaria Latinoamericana, Año 34, $\mathrm{n}^{\circ} 68$ (2008), p. 20 [consulté le 25/06/2021] <URL: http://www.jstor.org/stable/25479077>.

61. Carmen Boullosa, Cielos, Op. cit., p. 369.

\section{RÉSUMÉS}

Cielos de la Tierra (Carmen Boullosa, 1997) s'inscrit dans le double cadre de la métafiction historique et du roman d'anticipation. Trois narrateurs y dialoguent entre passé, présent et futur, dans une commune obsession pour la sauvegarde de la mémoire historique qui s'incarne dans le manuscrit d'Hernando de Rivas, intellectuel indigène du XVIe siècle.

L'élan humaniste de la Renaissance et des Lumières repose sur l'idée d'un accomplissement de l'homme par l'éducation et la culture, qui ne peut se réaliser que dans la confrontation avec le passé. C'est ce rapport au passé qui se trouve menacé dans les trois temps du récit, et plus particulièrement dans la ligne future, puisqu'au nom de l'état de perfection auquel elle aspire, la société atlante en vient à abjurer complètement la mémoire culturelle, avant d'abolir le langage et de s'engager dans une spirale autodestructrice.

On s'attachera à examiner l'articulation des ces trois temps et le problème de la transmission de la mémoire culturelle, qui interroge le devenir de l'humanisme à l'heure où émergent de nouvelles conceptions de l'homme : au seuil de la trans-humanité.

Cielos de la Tierra (Carmen Boullosa, 1997) is both a historiographic metafiction and a science fiction novel. Between past, present and future, the three narrators enter in a dialogue, united by a shared obsession with saving the historical memory embodied in the manuscript of Hernando de Rivas, a $16^{\text {th }}$ century native intellectual.

The humanistic impetus inherited from the Renaissance and the Enlightenment is based on the idea that man can improve through education and culture, which is made possible only by confronting the past. This relationship with the past is threatened in the three timelines of the story, and more specifically in the account of the future, since the Atlant society eventually renounces cultural memory, and then language, locking itself into a spiral of self-destruction.

The subject of this paper is the articulation between the three timelines and the transmission of cultural memory, which raises questions about the fate of humanism at a time when new conceptions of man are arising : on the threshold of transhumanity.

\section{INDEX}

Keywords : Carmen Boullosa, Cielos de la Tierra, historical Memory, Humanism, Transhumanism Mots-clés : Carmen Boullosa, Cielos de la Tierra, mémoire historique, humanisme, transhumanisme 
AUTEUR

MARIE-CAROLINE LEROUX

Université de Limoges 